\title{
Centrales fotovoltaicas: contexto colombiano frente a latinoamérica continental de habla hispana
}

\section{Photovoltaic Centrals: Colombian Context with Respect to Spanish Speaking Continental Latin America}

\author{
Diego Fernando Chacón Serna ${ }^{\star}$, Cristhian Mauricio Moreno Amaya ${ }^{\star \star}$, Johann \\ Alexander Hernández Mora ${ }^{\star \star \star}$ \\ Proyecto Curricular de Ingeniería Eléctrica, Facultad de Ingeniería Universidad \\ Distrital Francisco José de Caldas
}

FeCha DE ENTREGA: 24 DE NOVIEMBRE DE 2015

FECHA DE EVALUACión: 11 DE FEBRERo DE 2016

FECHA DE APROBACIÓN: 4 DE MARZO DE 2016

\begin{abstract}
Resumen Este artículo propone un análisis del sector fotovoltaico de cada uno de los países de habla hispana de América continental, haciendo un fragmento en regiones; Centro América y Sur América respectivamente. El crecimiento de la generación de energía eléctrica por medio de centrales fotovoltaicas ha tenido un gran impacto en el planeta y a su vez ha generado que en los últimos a ̃̂śos Latinoamérica tenga un crecimiento importante en el uso de esta tecnología para generar energía. Sin embargo, Colombia país que hace parte de América continental de habla hispana está atrasada en los avances que se han visto de manera general en Latinoamérica en cuanto a proyectos de gran escala, proyectos a pequeña escala y normatividad que regule e incentive el uso de esta tecnología para la generación de energía eléctrica.
\end{abstract}

\begin{abstract}
This article proposes a photovoltaic sector analysis of each one of the Spanish speaking countries of Continental America, by region fragmentation: Central and South America, respectively. The growth of electrical energy generation through photovoltaic centrals has had high impact on the planet and at the same time, has caused Latin America to increasingly use this technology during the last years to generate energy. However, Colombia (a country that is part of this group) is behind regarding the general progress shown in Latin America - with respect to large scale, small scale, and regulation projects that standardize and promote the use of this technology in order to generate electrical energy.
\end{abstract}

\footnotetext{
* diegounlited@hotmail.com

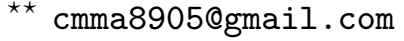

$\star \star \star$ jahernandezm@udistrital.edu.co
} 
Palabras Clave: central fotovoltaica, energía solar, radiación solar, incentivos, normatividad

Keywords: photovoltaic central, solar energy, solar radiation, incentives, regulation

\section{Introducción}

La generación mediante la tecnología solar, y su uso como fuente generadora de energía a gran escala mediante la figura de centrales y/o huertas fotovoltaicas ha experimentado un crecimiento exponencial en los últimos 20 años, destacándose principalmente el desarrollo en países de Europa y Norte América [1]. Este crecimiento se ha dado debido al desarrollo de políticas ambientales y la preocupación por temáticas como el cambio climático y otros efectos sufridos por el medio ambiente. Dichos aspectos han sido tratados desde la época del protocolo de Kioto y la Cumbre de Río ([2] a [4]). Por otra parte, la tecnología solar fotovoltaica ha demostrado traer consigo beneficios sociales y económicos en los lugares donde se ha implementado [5]. Todo lo anterior, sumado a la constante evolución de esta tecnología, reflejada en mejoras de rendimiento, de eficiencia y disminución de costos, ha hecho que sume cada vez más adeptos en todo el mundo $[6,7]$.

Por su parte, este sector en Latinoamérica ha empezado a mostrar incipientes crecimientos durante los últimos dos años ([1] a [8]). Consecuentemente, para lograr un adecuado desarrollo de la tecnología en los países latinos, se hace necesario conocer no sólo de las experiencias de sociedades maduras en el tema, sino también de las diferentes políticas y enfoques que se están dando en las propias. El presente trabajo propone un análisis del sector fotovoltaico de manera individual de cada país que comprende la América continental de habla hispana, y a su vez una segmentación por regiones (Centroamérica y Suramérica, respectivamente), para así poder realizar una comparación global de lo que sucede con respecto al tema de centrales fotovoltaicas en la zona, con la situación y medidas de Colombia al respecto. Se han considerado de forma sucesiva los siguientes asuntos: potencial energético, ámbito normativo, proyectos en funcionamiento y proyecciones futuras.

\section{Recurso de la energia solar fotovoltaica en los paises de habla hispana en América continental}

El recurso solar como base para la generación de energía eléctrica por medio de sistemas fotovoltaicos posee un gran potencial en América Latina, dadas sus condiciones geográficas, climáticas [9]. La figura 1 muestra un mapa de radiación global en la región tomado de Solar Gis Maps of Global Horizontal Irradiation Latin America. Adicionalmente, el cuadro 1 muestra un resumen del rango específico de radiación que se presenta en cada uno de los países analizados en éste estudio (con su respectiva fuente de información), los cuales se han denominado "países de habla hispana de América Continental". Es importante 
destacar que algunos presentan rangos más grandes que otros, lo cual se debe a factores como la extensión del país, las condiciones estacionales y por supuesto las condiciones de cada región particular.

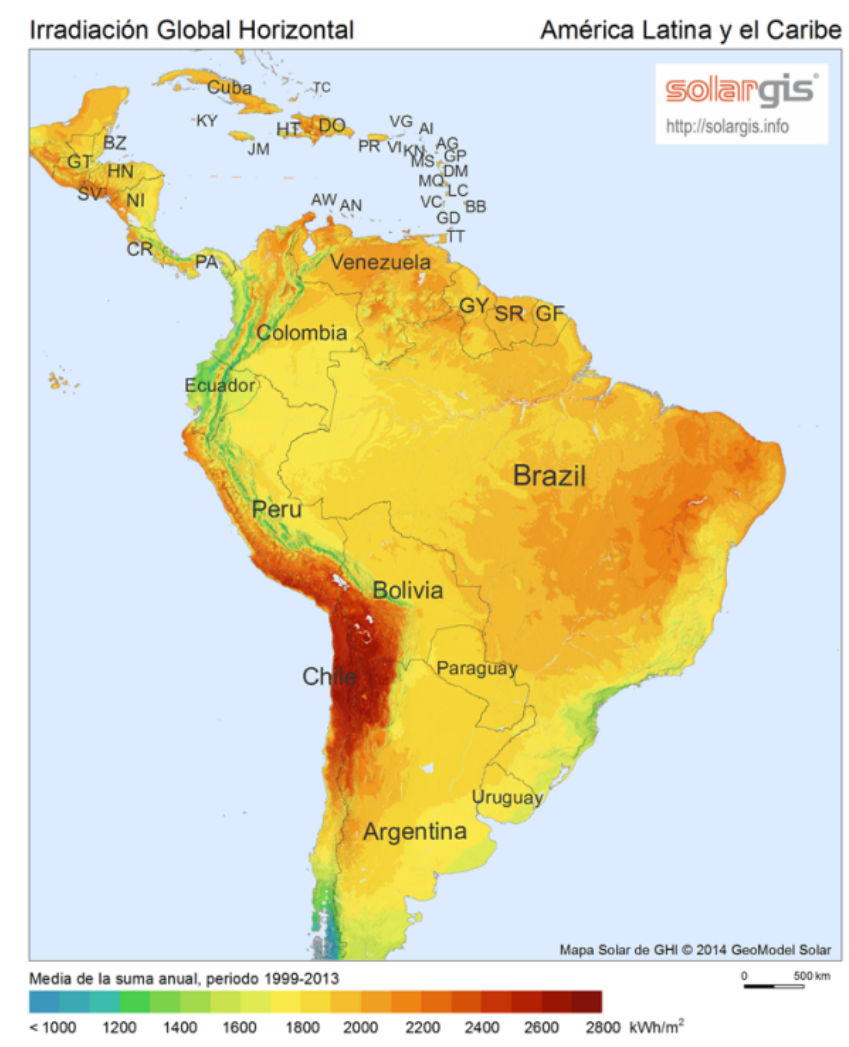

Figura 1. Mapa de irradiación Global Latinoamérica.

\section{Países de centroamérica continental de habla hispana y su visión y aplicación de centrales y o parques fotovoltaicos.}

\subsection{México}

El mercado eléctrico mexicano contempla diversas actividades, y cada una de estas tiene su respectiva normativa y regulación: generación, conducción, transmisión, transformación, distribución, abastecimiento, importación y exportación de energía. Dentro de algunas éstas (generación, transmisión, distribución y comercialización), se involucran tanto entes privados como públicos ([25] a [26]). La matriz de generación energética en México es muy variada según la tecnología que se usa, pero si se mira desde el punto de vista de los recursos que utilizan esta 


\begin{tabular}{lc}
\hline País & Rango de Radiación $\left[\mathrm{KWh} / \mathrm{m}^{2}\right]$ \\
\hline Argentina [10] & $2-7.5$ \\
Chile [11] & $3-7.5$ \\
Ecuador [12] & 4.2 \\
Guatemala [13] & $3.5-7.5$ \\
Honduras [14] & $3.5-6.2$ \\
México [15] & $4.4-6.3$ \\
Nicaragua [16] & $4-6.5$ \\
Perú [17] & $3.5-6.5$ \\
El Salvador [18] & $4-6$ \\
Uruguay [19] & $2.1-6.7$ \\
Venezuela [20] & $4-6$ \\
Panamá [21] & $3.8-6.9$ \\
Bolivia [22] & $4.2-6.3$ \\
Paraguay [23] & $3-6.5$ \\
Colombia [24] & $2.5-7$ \\
\hline
\end{tabular}

Cuadro 1. Rangos de radiaciones por país.

muy inclinado el uso de recursos no renovables con un $85 \%$ aproximadamente, mostrando su gran dependencia a estas fuentes. Por otra parte, están los recursos renovables, los cuales podrían sumar ese $15 \%$ restante, distribuido en centrales hidroeléctricas y geotérmicas principalmente, tal como se evidencia en [27]. De acuerdo con lo anterior, el país ha estado generado una política de incentivos [27], enfocada en fomentar el empleo de nuevos recursos renovables dentro de los cuales se destaca la tecnología solar fotovoltaica. Adicionalmente, dentro del país se cuenta con sector maduro empresas de buena experiencia en el área.

México, adicional a las buenas condiciones de radiación que presenta, ha logrado a la fecha una capacidad de producción anual de módulos superior a los 312 MW [28], que además de ser una gran producción desde el punto de vista económico interno haría muy viable la aplicación e implementación de esta tecnología ya que no se tendría que importar en exceso si no se podría manejar en el mercado interno mexicano, lo que se ha dado como consecuencia la ley para el aprovechamiento de energías renovables y el financiamiento de la transición energética [29]. En el cuadro 2 se describen los proyectos identificados en México ([30] a [35]).

\subsection{Guatemala}

Guatemala es uno de los países de América central que está impulsando el uso de los recursos naturales como fuentes no convencionales para la generación de energía eléctrica. Existen varios proyectos que se están realizando actualmente mediante cooperación entre el gobierno de Guatemala y entidades privadas. Adicionalmente, 


\begin{tabular}{lc}
\hline Proyecto & Potencia \\
\hline Aura Solar I & $39 \mathrm{MW}$ \\
Tai Durango I & $16 \mathrm{MW}$ \\
Central PV Cerro Prieto & $5 \mathrm{MW}$ \\
Parque Solar Bicentenario & $2 \mathrm{MW}$ \\
Central Solar Santa Rosalia & $1 \mathrm{MW}$ \\
\hline
\end{tabular}

Cuadro 2. Proyectos México.

los recursos extranjeros que llegan como inversión directa, pueden aprovechar los diferentes incentivos que decreta la Ley de Incentivos para el Desarrollo de Proyectos de Energía Renovables [36]. En el 2003 en Guatemala, entro en vigencia [36], en la cual el gobierno de Guatemala busca promover de forma activa, el aprovechamiento de los recursos naturales para la generación de energía eléctrica que permita a largo plazo, obtener un desarrollo continuo de dichas energías, con el fin de mejorar la calidad ambiental del país y llamar la atención de inversionistas en este sector. La responsabilidad del desarrollo de actividades, fomentos y utilización de las energías renovables es del Ministerio de Energía y Minas, el cual está encargado de la promoción y localización de los recursos para la generación de energía eléctrica, de impulsar los estudios para estimar el potencial técnico utilizable, de fomentar y facilitar el uso de inversiones para el desarrollo de proyectos, favoreciendo la oferta eléctrica nacional a través del uso de energías renovables y un menor uso de combustibles importados, y de facilitar los certificados para los proyectos de éste tipo que se establezcan en el país [37]. En el cuadro 3 se identifican los principales proyectos solares fotovoltaicos que actualmente se encuentran ya en funcionamiento [38,39].

\begin{tabular}{lc}
\hline Proyecto & Potencia \\
\hline Planta solar Fotovoltaica Horus I & $50 \mathrm{MW}$ \\
La Planta Fotovoltaica Sibo & $5 \mathrm{MW}$ \\
\hline
\end{tabular}

Cuadro 3. Proyectos Guatemala.

\subsection{El Salvador}

El Salvador es un país en el cual se maneja un concepto de apertura en el mercado eléctrico en todos sus sectores (generación, transmisión y distribución), en los cuales se observa en gran medida la participación privada. En el sector de generación, Las fuentes renovables juegan un papel fundamental en el fortalecimiento 
y crecimiento de la matriz energética. El CNE (Consejo Nacional de Energía), tiene como política y prioridad el desarrollo sostenible mediante las tecnologías limpias y renovables [40]. En la Ley de Incentivos Fiscales para el Fomento de las Energías Renovables en la Generación de Electricidad [41] se contemplan excepciones fiscales de acuerdo a su capacidad del proyecto. No existe límite de capacidad pero si hay una división en tres categorías, tal como lo establece el Acuerdo No 162 - E-2012 [42]; en el cual se aprueba la "Normativa Técnica para Caracterizar los Proyectos que Aprovechan las Fuentes Renovables en la Generación de Energía Eléctrica" el cual va ligado con la ley 462 anteriormente citada: "... Artículo 6: De acuerdo al nivel de potencia o capacidad instalada de las centrales de generación eléctrica y para los fines de aplicación de esta norma, se establecen las siguientes categorías de proyectos: a) Menores de 10 megavatios $(M W)$; b) Entre 10 y 20 megavatios $(M W)$; y, c) Mayores de 20 megavatios $(M W) "[41]$.

Hacia abril de 2013, El Salvador había explotado el recurso solar fotovoltaico en pequeñas cantidades en sistemas autónomos aislados y algunos conectados a la red para autoconsumo en un número limitado de construcciones y cantidad de potencia, pero para los meses de diciembre de 2013 se puso en funcionamiento la primera planta conectada al sistema eléctrico, mediante una iniciativa llevada a cabo por la empresa RTS Energy, para mirar el comportamiento y la soportabilidad del sistema. Para estos momentos, El Salvador empieza a apostar por esta tecnología para diversificar su matriz energética, lo que hoy se ve reflejado en los principales proyectos mostrados en el cuadro 4 [43,44].

\begin{tabular}{lc}
\hline Proyecto & Potencia \\
\hline La Planta Fotovoltaica Esperanza & $2,88 \mathrm{MW}$ \\
La Planta Fotovoltaica PV Salvador & $70 \mathrm{MW}$ \\
\hline
\end{tabular}

Cuadro 4. Proyectos El Salvador.

\subsection{Honduras}

Honduras se encuentra ubicada en el centro de Centroamérica. A pesar de su situación económica, el país cuenta con amplias fuentes de energías renovables, principalmente de origen hídrico, aunque debido a su posición geográfica, también posee un no despreciable potencial eólico, solar y geotérmico [45]. El cuadro 5 muestra un resumen de los principales proyectos en funcionamiento en el país ([40] a [52]).

La proyección, los estudios y la puesta en marcha de estos proyectos se han podido realizar gracias a que Honduras cuenta con una normatividad que incentiva el uso de energías renovables, donde se destaca principalmente el Decreto 267-98, que reformó la Ley de incentivos emitida en abril de 1998 [53]. 


\begin{tabular}{lc}
\hline Proyecto & Potencia \\
\hline Planta Solar Pavana & $24 \mathrm{MW}$ \\
Planta Solar Marcovia Fase I & $35 \mathrm{MW}$ \\
Aura Solar II & $61 \mathrm{MW}$ \\
Agua Fría & $60 \mathrm{MW}$ \\
Nacaome & $73,2 \mathrm{MW}$ \\
Valle & $73,2 \mathrm{MW}$ \\
Fotersa & $70 \mathrm{MW}$ \\
\hline
\end{tabular}

Cuadro 5. Proyectos Honduras.

El objetivo de este decreto es incentivar el uso de las energías renovables para lo cual brinda unos beneficios como son: a) Exoneración de pago de impuestos sobre ventas (en la construcción), b) pago de los impuestos, tasas y derechos de las importaciones (durante los estudios y construcción), c) exoneración de impuestos sobre la renta (durante los 5 primeros años de operación).

Adicionalmente, existe una actualización mediante la Ley de Promoción a la Generación de Energía Eléctrica con recursos renovables, el decreto 70-2007 [54]. Mediante de este decreto se ratifica el apoyo del gobierno para el aprovechamiento de las energías renovables como fuente de generación de energía eléctrica. Este decreto consolida las exoneraciones de pago de impuestos y demás beneficios que en el decreto 267-98 se habían impuesto, y agregan otros beneficios adicionales tales como "La dispensa del pago de impuestos por importación temporal". Aparte de esto se menciona que las plantas de energía renovables recibirán por parte de la Empresa Nacional de Energía Eléctrica (ENEE), un contrato y por diez (10) años una tarifa $10 \%$ mayor que el costo marginal de corto plazo.

En mayo del 2014, se publicó el Decreto: Ley General Para la Industria Eléctrica [55] en la que se mencionan partes importantes para el uso y aprovechamiento de las energías renovables como fuente de generación de energía eléctrica. Como se ve, el uso de las energías renovables, entre ellas la Energía solar como fuente de generación de Energía eléctrica, ha tenido un fuerte apoyo por parte del gobierno y de las entidades competentes, quienes se encargan de regular, normalizar e incentivar el uso de este tipo de energías para tal propósito.

\subsection{Nicaragua}

El sector eléctrico nicaragënse ha presentado una gran evolución desde que en 1998 se empezó a generar la apertura al sector privado, inicialmente en transmisión y distribución, y luego como era de suponerse, en el sector de generación, el cual está basado en gran parte en centrales térmicas. A pesar del interesante potencial con el que cuenta este país, son muy pocos los avances en el sector debido a la falta de estudios realizados sobre el particular. En los últimos años se ha demostrado interés por parte del gobierno para aumentar el uso de las energías renovables y su 
viabilidad mostrada [56,57], pero el aspecto normativo no colabora mucho ya que la ley 532 "Para la Promoción de Generación Eléctrica con Fuentes Renovables" [58] es muy vaga y en el aspecto de las tecnologías fotovoltaicas en las diversas escalas de generación, no se dictan condiciones favorables para un desarrollo pronto de esta tecnología. Además, en el campo regulatorio para estas centrales falta fijar los límites de generación para así poder determinar a qué incentivo o incentivos se puede acceder. A pesar de estas falencias en la parte normativa y regulatoria, gracias a la inversión mayormente extranjera, Nicaragua ya cuenta con una central fotovoltaica llamada Darimba (cuadro 6), ubicada en la zona de la central del país [59].

\begin{tabular}{lr}
\hline Proyecto & Potencia \\
\hline Central Fotovoltaica Darimba. & $1,38 \mathrm{MW}$ \\
\hline
\end{tabular}

Cuadro 6. Proyectos Nicaragua.

\subsection{Costa Rica}

La filosofía energética en Costa Rica está basada en las fuentes renovables de energía, teniendo como su fuente principal la producción de energía mediante las hidroeléctricas, aunque con una apertura a que todas las fuentes renovables de energía que puedan ser utilizadas, para así poder satisfacer las necesidades de la población costarricense y además solidificar y dar un grado de fortaleza y robustez a la matriz energética [60]. La energía solar fotovoltaica como fuente de energía es muy bien vista, ya que desde el punto de vista del ICE (Instituto Costarricense de Electricidad), el país cuenta con zonas de alto potencial solar, la tecnología ha tenido un gran desarrollo y sus costos han disminuido [61]. Además, las otras tecnologías han incrementado costos y tienen implicaciones socio ambientales son mucho más complejas en cuanto a construcción e instalación.

El marco normativo de fuentes renovables no convencionales se encuentra dentro de la ley "Generación Eléctrica Paralela o Autónoma" [62], en donde incluyen a los parques fotovoltaicos y/o centrales fotovoltaicas. Estas se encuentran como centrales de capacidad limitada que pueden ser operadas por un agente privado o una cooperativa de electrificación rural. Además la ARESERP (Autoridad Reguladora de los Servicios Públicos) estimó hace un año aproximadamente que la tarifa que se aplicaría a la energía fotovoltaica sería similar a la energía hidráulica sabiendo de antemano que la energía hidráulica maneja una franja de tarifas y el precio se deberá negociar con la Distribuidora o directamente con el ICE [63].

Actualmente, en Costa Rica una de las formas de utilizar la tecnología solar fotovoltaica es para autoconsumo, ya que se permite la venta de excedentes e 
intercambios de los mismos [62]. También, para noviembre de 2012 entró en funcionamiento la primera central llamada Parque Solar Miravalles (cuadro 7), y tiene como proyecciones cercanas 2 centrales de mayor capacidad (5 MW) que saldrán a la venta propuestas por el ICE [64].

\begin{tabular}{lc}
\hline Proyecto & Potencia \\
\hline Parque Solar Miravalles & $1 \mathrm{MW}$ \\
\hline
\end{tabular}

Cuadro 7. Proyectos Costa Rica.

\subsection{Panamá}

El mercado eléctrico panameño se caracteriza por los sectores ya conocidos generación, transmisión y distribución. Cada uno de éstos tiene características principales, y se separa en regulado y no regulado. Los sectores de la transmisión y distribución hacen parte de los regulados, y la generación de gran interés no tienen regulación, lo que hace inferir que hay libertad de precios, costos variables auditables y se puede generar venta por medio de contratos o en mercados ocasionales, todo esto bajo la responsabilidad del CND (Centro Nacional de Despacho) [65] [66]. La generación, como en otros países de la región se basa en tecnología hídrica, seguida por la térmica. Sin embargo, también es un país que muestra sumo interés por diversificar la matriz energética con fuentes renovables [66] [67], mediante centrales eólicas y fotovoltaicas. Ya existe en funcionamiento el Parque Solar Sarigua de 2,4 MW, y se prevé un potencial en proyectos con licencias definitivas entre 300MW y 350 MW, lo cual ayudaría a la diversificación de la matriz y a dar un soporte en temporadas de sequía. Además, se están promulgando leyes de incentivos para las energías renovables por separado, según la tecnología. En el caso de la solar fotovoltaica, la ley 37 "Establece el régimen de incentivos para el fomento de la construcción, operación y mantenimiento de centrales y/o instalaciones solares" [68]. En esta ley, la centra fotovoltaica se establece como la instalación de generación superior a $500 \mathrm{~kW}$ de capacidad instalada. El cuadro 8 describe los proyectos que ya se encuentra en funcionamiento y los proyectados [69]:

\section{Países de suramérica de habla hispana y su visión y aplicación de centrales y/o parques fotovoltaicos.}

\subsection{Argentina}

El mercado eléctrico argentino, debido a la cantidad de coyunturas y problemáticas que ha vivido el país [70], es de participación mayoritariamente privado en todas 


\begin{tabular}{lc}
\hline Proyecto & Potencia \\
\hline Parque Solar Sarigua & $2,4 \mathrm{MW}$ \\
Licencia Definitiva & \\
Avanzalia Panama & $120 \mathrm{MW}$ \\
Panama Solar Energy Providers & $45 \mathrm{MW}$ \\
SDR Energy Panama & $49,5 \mathrm{MW}$ \\
Solar XXI & $40 \mathrm{MW}$ \\
Otros (menores $(20 \mathrm{MW}))$ & $89.592 \mathrm{MW}$ \\
\hline
\end{tabular}

Cuadro 8. Proyectos Panamá.

las actividades (generación, transmisión, distribución). Sin embargo estas están estrictamente ligadas a las normativas del gobierno y los entes estatales de regulación. Así mismo, es un mercado que centraliza sus esfuerzos hacia el Gran Buenos Aires que es lugar de mayor concentración poblacional y por ende el de mayor demanda del suministro de energía eléctrica [71]. En la parte de generación, es un país basado en las fuentes térmicas, basadas a gas natural, principalmente importado de Bolivia, y dependiente de otros combustibles fósiles en el aspecto térmico. También cuenta con generación hidroeléctrica, nuclear a pequeña escala y en cuanto a fuentes de energía renovables no convencional se encuentra muy limitada su participación en la matriz energética del país. Por eso desde años recientes surgió la necesidad y la idea, bajo el programa GENREN, de diversificar la matriz de generación por parte de las fuentes renovables [72], la meta a 2019 es que el $8 \%$ de la generación total del país la aporten estas fuentes, donde entraría a participar las centrales fotovoltaicas como opción y aplicación para satisfacer esta y otras metas futuras. Inicialmente, GENREN proponía 20MW licitados para la parte solar fotovoltaica y fue modificada a $10 \mathrm{MW}$ años posteriori, debido a la visión certera de la incapacidad de poder cumplirla. Gracias a la inversión privada y a los planes de cada una de las diferentes provincias en sus planes regionales energéticos, es posible decir que en la parte solar fotovoltaica la meta se sobrepasaría. Además, se ve una gran proyección en el país como causa y consecuencia de la instalación y ampliación en producción de diversas fábricas de módulos fotovoltaicos, lo que hace presumir un alza en el mercado de esta fuente energética en la zona [72]. La parte normativa y legal para este aspecto está regida por la ley 26.190 "Régimen de Fomento Nacional para el uso de Fuentes Renovables de Energía Destinada a la Producción de Energía Eléctrica" [73], en la cual se plantean una serie de incentivos y beneficios que se obtendrían al invertir en estas fuentes. En el cuadro 9 se identifican algunos de los proyectos en Argentina ([74] a [77]). 


\begin{tabular}{lc}
\hline Proyecto & Potencia \\
\hline Chimbera 1 & $2 \mathrm{MW}$ \\
Parque Fotovoltaico San Juan & $1,2 \mathrm{MW}$ \\
Cañada Honda & $5 \mathrm{MW}$ \\
Terrazas del Portezuelo & $1 \mathrm{MW}$ \\
\hline
\end{tabular}

Cuadro 9. Proyectos Argentina.

\subsection{Perú}

El mercado eléctrico peruano ha estado a la altura del crecimiento y desarrollo económico del país. Según el Ministerio de Energía y Minas (MIMEM) de Perú, la capacidad de generación total instalada conectada al Sistema Eléctrico Interconectado Nacional (SEIN) en 2013 consistía principalmente en 23 centrales hidroeléctricas con una capacidad de $3.270 \mathrm{MW}$ y 32 centrales termoeléctricas con una capacidad total de 5.260 MW. Como consecuencia de la subasta de energías renovables, cuatro (4) plantas solares con una capacidad total de generación de 80 MW también estaban en operación en el 2013 [78].

En Perú existen dos instrumentos legales que establecen el marco legal para el sector eléctrico procedente de energías no convencionales: el primero es el Decreto Legislativo No 1002 de promoción de la inversión para la generación de electricidad con el uso de energías renovables [79], y el segundo es el reglamento de generación de electricidad con energías renovables, Decreto Supremo 050-2008-EM [80]. Este último establece las normativas para la promoción de los recursos de energía renovables como prioridad nacional y fomenta la conexión de la electricidad procedente de energías renovables. En este también se establece una máxima cuota quinquenal de la demanda de energía que debe satisfacerse con electricidad procedente de los recursos de energías no convencionales. Adicionalmente, también se establecen los incentivos fiscales para el uso de las energías renovables, los cuales consisten en que toda la generación de electricidad que se realice a partir de fuentes no convencionales tiene derecho a una amortización acelerada del $20 \%$ sobre el impuesto de renta. Esta amortización acelerada aplica para los equipos, maquinarias, instalaciones y operación de las centrales con generación de energía renovables, y los generadores tienen derecho al reembolso antes de plazo del impuesto al valor agregado (IVA) por la venta de electricidad.

Por otra parte, el segundo instrumento que existe en Perú como normativa aplicada a las energías renovables es la Norma Técnica Peruana (NTP) [81], que establece la regulación de los equipos de energía renovables para sistemas solares fotovoltaicos de hasta $500 \mathrm{Wp}$ de potencia, pero este instrumento aplica para instalaciones domiciliarias. El cuadro 10 describe los proyectos solares fotovoltaicos que se encuentran en operación y los que están proyectados ([82] a [90]). 


\begin{tabular}{lc}
\hline Proyecto en Operación & Potencia \\
\hline Panamericana Solar & $20 \mathrm{MW}$ \\
Tacna Solar & $20 \mathrm{MW}$ \\
Majes Solar & $20 \mathrm{MW}$ \\
Proyectados & \\
Central Solar Arepa & $30 \mathrm{MW}$ \\
Central Solar La Joya & $100 \mathrm{MW}$ \\
Central Solar Pampa Colorada & $100 \mathrm{MW}$ \\
Central Solar Pampa Las Pulgas & $40 \mathrm{MW}$ \\
Central Solar Rampa Siguas & $40 \mathrm{MW}$ \\
Central Solar Tesoro & $40 \mathrm{MW}$ \\
\hline
\end{tabular}

Cuadro 10. Proyectos Perú.

\subsection{Ecuador}

El mercado eléctrico ecuatoriano se ha caracterizado por tener tanto intervención estatal como privada en los diferentes sectores del mercado, como lo son la generación y comercialización, con un ente regulador estatal (CONELEC). En generación, su matriz energética está principalmente dividida en partes muy proporcionadas entre la tecnología térmica e hídrica [91]. En los últimos años se ha emprendido la política de fomentar implementar y explotar los recursos renovables y dejar la dependencia por parte del sector térmico, ya que los combustibles para estos generadores son generalmente importados, lo cual generan un gran impacto económico en el país [92]. Inicialmente, por medio de las fuentes eólica y geotérmica se generaron varios proyectos. Igualmente, se esperaba que sucediera lo mismo con la tecnología solar fotovoltaica, pero al darse una gran inversión estatal en megaproyectos hidroeléctricos esto ha sido pausado, y lo que se ha emprendido es una ampliación en la generación hídrica. En el cuadro 11 están los proyectos constituidos como parque, planta y/o central Fotovoltaica ([93] a [95]).

\begin{tabular}{lc}
\hline Proyecto & Potencia \\
\hline Planta Solar Fotovoltaica Santa Elena & $25 \mathrm{MW}$ \\
Planta Solar Tierra del Sol & $28 \mathrm{MW}$ \\
Salinas & $1 \mathrm{MW}$ \\
Tren de Salinas & $2 \mathrm{MW}$ \\
\hline
\end{tabular}

Cuadro 11. Proyectos Ecuador. 


\subsection{Uruguay}

Como es característico en la región, el sector eléctrico uruguayo ha sufrido un proceso de desintegración vertical del mercado, y dentro de su propio mercado se ha creado un ámbito de libre competencia en las actividades de generación y comercialización de energía eléctrica, fijando el rol del estado como ente regulador y administrador del mercado [96]. En la parte de la generación, como es característico en la región del rio de la plata, hay una gran división en las fuentes de generación casi proporcional en generación hídrica y térmica [96]. En esta última hay gran dependencia de los combustibles fósiles y una pequeña participación los combustibles de la biomasa. En cuanto a política energética, el país también optado por busca diversificar la matriz energética mediante fuentes renovables no convencionales [97] [98]. Adicionalmente, mediante el Plan Solar [99] se busca concientizar a la población de Uruguay con respecto a la eficiencia energética. Todo esto incentivado por la Ley de Promoción y Protección de Inversiones [100]. A la fecha, Uruguay cuenta con el siguiente proyecto del cuadro 12 [101].

\begin{tabular}{lc}
\hline Proyecto Aprobado & Potencia \\
\hline Proyecto Energía Solar La Jacinta & $65 \mathrm{MW}$ \\
\hline
\end{tabular}

Cuadro 12. Proyectos Uruguay.

\subsection{Bolivia}

El sector eléctrico boliviano, como en todos los países que se han mencionado, cuenta con una Ley referente a la generación, transmisión y distribución de energía eléctrica: Ley No 1604 de Electricidad de 1994 [102]. En el Artículo 5 de esta Ley se contempla el aprovechamiento de los recursos naturales, en los que se menciona el agua y otros recursos naturales renovables destinados a la producción. Sin embargo, no hay mucho que decir de esta Ley en lo que respecta a la regulación, normatividad y promulgación de incentivos para promover el uso de energías renovables como fuente de energía para la generación de energía eléctrica. Esto sucede ya que más del $50 \%$ de la generación se realiza por medio de centrales termoeléctricas a base de carbón, y el restante por medio de centrales hidroeléctricas. Por otra parte, existe un plan gubernamental de electrificación solar enfocado únicamente a proyectos a pequeña escala, aislados con fines residenciales para las zonas que presentan difícil acceso, o zonas no interconectadas [103]. A pesar de que no existe alguna regulación, Bolivia cuenta con un proyecto solar fotovoltaico (cuadro 13) [104]. 


\begin{tabular}{lc}
\hline Proyecto & Potencia \\
\hline Planta Solar Pando $5 \mathrm{MW}$ \\
\hline Cuadro 13. Proyectos Bolivia.
\end{tabular}

\subsection{Paraguay}

El país Paraguayo, se caracteriza por tener una única empresa eléctrica integrada verticalmente: ANDE [105]. El parque generador del Sistema Eléctrico Nacional está conformado por dos Centrales hidroeléctricos: Itaipú y Yaciretá, ambas de carácter binacional, por la Central Hidroeléctrica Acaray que es de carácter nacional, y algunos grupos pequeños de Centrales Térmicas [106]. En su marco político, contempla la Ley 3009 de la producción y transporte independiente de energía eléctrica [107], en la cual se establece la producción independiente de energía eléctrica a partir de fuentes no convencionales de energía. Sin embargo, para esta Ley no se contempla algún decreto reglamentario relacionado con incentivos para la promulgación de la generación de energía eléctrica con fuentes no renovables. Este país no cuenta con algún proyecto solar fotovoltaico aprobado o en proyección.

\subsection{Venezuela}

Venezuela es un país en el cual la generación de energía eléctrica está conformada por un $62 \%$ con Centrales Hidroeléctricas, un $35 \%$ con Centrales Térmicas y un $3 \%$ corresponde a Centrales de generación distribuida, conformada por grupos electrógenos [107]. El país Venezolano no cuenta con una Ley regulatoria para la aplicación de la generación de energía eléctrica por medio de fuentes de energías renovables no convencionales. Sin embargo se dice que el estado Venezolano ha estado impulsando varias acciones de fomento y desarrollo de las energías renovables, en la cual se contempla la construcción de dos parques eólicos ubicados en la parte costera noroccidental, con una capacidad de generación de $200 \mathrm{MW}$ [108] . En el mismo contexto, en el ámbito de la energía solar fotovoltaica, existe un programa llamado Sembrando Luz, el cual está destinado a proveer de energía eléctrica a zonas aisladas y no interconectadas [109]. Aparte de estos proyectos, Venezuela no cuenta con más proyectos solares fotovoltaicos existentes.

\subsection{Chile}

En la década de los 40 Chile enfrentó la necesidad de electrificar el país, tema que lo solucionó con la creación de ENDESA [110]. De esta manera fue como los sistemas aislados fueron constituyendo lo que se conoce como el sistema interconectado del norte grande (SING) [111] y del central (SIC) [112]. A partir de esto, se lleva a cabo la desintegración vertical del sector, separando las actividades 
de generación, transmisión y distribución de energía eléctrica. A diciembre de 2012, el SIC sumaba una potencia instalada de 13.454 MW, y cuenta con las tres cuartas partes de la potencia instalada a nivel nacional y abastece a más del $90 \%$ de la población. Por su parte el SING suma una potencia instalada de 4.581 MW, y cuenta con una cuarta parte de la potencia instalada a nivel nacional, y a diferencia del resto del país, está constituida casi exclusivamente por centrales termoeléctricas. Estos dos sistemas entregan una capacidad instalada total de 18.277 MW, lo que descontando de sus consumos propios, constituye una capacidad neta instalada alrededor de los 17.000 MW. En la edición 39.525 del Diario Oficial de la República de Chile del 1 de diciembre de 2009 [113], se crea el comité de asignación de fondos a energías renovables no convencionales. La parte normativa y legal está regida para este aspecto por la Ley 20257 Ley general de servicios eléctricos respecto de la generación de energía eléctrica con fuentes de energías renovables no convencionales [114]. En el cuadro 14 están los proyectos en operación y en fase de construcción ([115] a [125]).

\begin{tabular}{lc}
\hline Proyectos en construcción & Potencia \\
\hline Ravsoe Planta Solar Fotovoltaica & $37 \mathrm{MW}$ \\
Pampa Solar Norte PV & $91 \mathrm{MW}$ \\
El Tamarugo Solar Granja & $22 \mathrm{MW}$ \\
El Chañar Solar & $22 \mathrm{MW}$ \\
Laberinto Solar & $72 \mathrm{MW}$ \\
Sol De Loa & $110 \mathrm{MW}$ \\
Chaka PV & $20 \mathrm{MW}$ \\
Complejo Solar PV & $90 \mathrm{MW}$ \\
Huerta Solar PV & $8 \mathrm{MW}$ \\
Proyectos en operación & \\
Maria Elena & $72 \mathrm{MW}$ \\
Amanecer & $100 \mathrm{MW}$ \\
\hline
\end{tabular}

Cuadro 14. Proyectos Chile.

\section{Comparación de los marcos regulatorios para la energía solar fotovoltaica en los países de habla hispana del continente americano}

En esta sección se muestra una visión general y descriptiva del marco normativo y/o regulatorio de cada país y su influencia en el desarrollo de proyectos de centrales fotovoltaicas. Se presenta un breve análisis de lo que sucede en el marco normativo de cada país en el aspecto renovable y por ende influye en el concepto 
de centrales fotovoltaicas, para así poder identificar tendencias normativas y/o regulatorias por región, e identificar porque hay países con mayor inversión y surgimiento en esta tecnología que otros.

\subsection{México}

Ley para el Aprovechamiento de Energías Renovables y el Financiamiento de la Transición Energética.

- Se hace una diferenciación entre las fuentes con capacidad superior a los 2,5 MW y las que no, donde las de mayor capacidad adquirirán compromisos sociales con las comunidades cercanas al proyecto.

- No se establece un límite de capacidad para las diversas fuentes incluidas en esta ley exceptuando las fuentes hídricas, superiores a 30 MW.

- Se busca la reducción de la dependencia de México de los hidrocarburos como fuente primaria de energía.

- Se crea el Fondo para la transición Energética y el Aprovechamiento Sustentable de la Energía; con el propósito de potenciar el financiamiento e inversión en las Energías Limpias.

\subsection{Guatemala}

Ley de Incentivos para el Desarrollo de Proyectos de Energía Renovable.

- Todas las fuentes renovables tienen derecho a ciertos incentivos desde que cumplan los requerimientos por ley, y cumplan el proceso de evaluación y clasificación del proyecto.

- Los incentivos se identifican en el decreto 52-2003, los cuales son:

1. Exención de derechos arancelarios para las importaciones, iva.

2. Exención del pago del Impuesto Sobre la Renta. Durante un periodo de 10 años.

3. Exención del Impuesto a las Empresas Mercantiles y Agropecuarias. Durante un periodo de 10 años.

\subsection{El Salvador}

Ley de Incentivos Fiscales para el Fomento de las Energías Renovables en la Generación de Electricidad.

- Se establecen los siguientes beneficios e incentivos fiscales:

1. Exención del DAI (Derechos Arancelarios de importación) durante 10 Años para proyectos de hasta $20 \mathrm{MW}$.

2. Exención del pago de renta, durante 5 años a proyectos entre 10 y 20 MW; y 10 años a proyectos menores a 10 MW.

3. Exención total de pago de impuestos sobre los ingresos provenientes de las ventas, Reducciones Certificadas de Emisiones. 
4. Proyectos superiores a 20 MW tendrán deducción de pago de renta, durante máximo 10 años.

5. Deducción en créditos fiscales.

- Se caracterizaran los proyectos en: Menores a 10 MW, Entre 10 y 20 MW, y Mayores a $20 \mathrm{MW}$.

\subsection{Honduras}

Ley de Incentivos; Ley de Promoción a la Generación de Energía Eléctrica con recursos renovables. (Decreto 267-98; Decreto 70-2007)

- Exoneración de pago de impuestos sobre ventas (en la construcción).

- Pago de los impuestos, tasas y derechos de las importaciones (durante los estudios y construcción).

- Exoneración de impuestos sobre la renta (durante los 5 primeros años de operación).

\subsection{Nicaragua}

Ley No. 532: Ley para la Promoción de la Generación Eléctrica con Fuentes Renovables. Se establecen los siguientes beneficios con tal de promover las fuentes renovables:

1. Exoneración del pago de los Derechos Arancelarios de Importación (DAI).

2. Exoneración del pago del IVA.

3. Exoneración de impuesto sobre la Renta.

4. Exoneración de todos los impuestos municipales vigentes.

5. Exoneración de todos los impuestos que existan por explotación de riquezas por un periodo de 5 años.

6. Exoneración del impuesto Timbres Fiscales (ITF).

\subsection{Costa Rica}

Ley 7200: Ley que Autoriza la Generación Eléctrica Autónoma o Paralela.

- Se establece el límite de capacidad a las fuentes no convencionales de energía el cual es $20 \mathrm{MW}$.

- Se tendrán exenciones fiscales en la importación de maquinaria.

\subsection{Panamá}

Ley 37: Ley que Establece el Régimen de Incentivos para el Fomento de la Construcción, operación y mantenimiento de centrales y/o Instalaciones Solares. Diferencia las centrales fotovoltaicas en, Pequeñas Centrales Particulares De Energía Solar cuya capacidad será de 500 MW máximo y Sistemas de Centrales Solares cuya capacidad es superior a 500 MW. Se establecen una serie de incentivos para el uso de esta tecnología los cuales son: 
1. Exoneración de impuestos por importación.

2. Crédito fiscal aplicable sobre la renta liquidada en la actividad en un periodo fiscal determinado, por un máximo del $5 \%$, del valor total de la inversión.

3. Utilización del método de depreciación acelerada.

\subsection{Ecuador}

Regulación No Conelec-001/13 Define el uso de la energía solar fotovoltaica, mas no estipula límites, incentivos con respecto a esta fuente de generación. Venezuela. Carece de normativas y leyes para la producción de energías alternativas.

\subsection{Perú}

Decreto Legislativo No 1002: Decreto Legislativo De Promoción De La Inversión Para La Generación De Electricidad Con El Uso De Energías Renovables. Se planteó con el fin de mostrar el interés por el desarrollo de nuevas fuentes mediante recursos renovables, y así mejorar la calidad de vida de la población y proteger al medio ambiente, promoviendo el uso de estas nuevas tecnologías, mediante los siguientes decretos:

- D.S No 012-2011-EM, aprueba el reglamento de generación de electricidad con energías renovables.

- D.S No 020-2013-EM, aprobó el reglamento para la promoción de la inversión eléctrica en áreas no conectadas a red.

\subsection{Bolivia}

Carece de normativas y leyes para la producción de energías alternativas.

\subsection{Uruguay}

Ley 16.906 Ley de Promoción y Protección de Inversiones. (Decreto No 354/009) En la cual se soporta los beneficios de inversión y beneficios fiscales que tendrán las actividades que sean destinadas al sector productivo como lo es la generación eléctrica, dando cabida a la inversión privada, pública o mixta, mediante los siguientes decretos:

- Decreto No 113-013, donde se considerara a efectos del despacho las centrales fotovoltaicas con un costo variable.

- Además se hace una división por franjas explicada a continuación:

1. Franja1: $500 \mathrm{MW}$ a $1000 \mathrm{MW}$.

2. Franja2: $1 \mathrm{MW}$ a $5 \mathrm{MW}$.

3. Franja3: 5 MW a 50 MW.

- Además se encuentran decretos directamente asociados para la energía solar fotovoltaica, los cuales son:

1. Decreto $173 / 010$ para micro generación.

2. Resolución 1895/010 para bajas potencias.

3. Resolución 1895/010 para altas potencias. 


\subsection{Paraguay}

vLey 3009: De la Producción y Transporte Independiente de Energía Eléctrica. Establece a producción independiente de energía eléctrica a partir de fuentes no convencionales de energía.

\subsection{Argentina}

Ley 26.190: Régimen de Fomento Nacional para el uso de Fuentes Renovables de Energía Destinada a la Producción de Energía Eléctrica. Establece como objetivo alcanzar un $8 \%$ de participación de las energías renovables en el país. Se contemplan algunos beneficios arancelarios como:

1. Devolución anticipada y amortización acelerada dispuestos en la ley 25924 .

2. Exenciones en IVA, e impuesto a las ganancias; dispuesto en la ley 25063.

\subsection{Chile}

Ley 20.25\%. Introduce Modificaciones A La Ley General De Servicios Eléctricos Respecto De La Generación De Energía Eléctrica Con Fuentes De Energías Renovables No Convencionales. No establece límites de capacidad, pero si la capacidad es mayor a 200 MW para comercializar o distribuir con el cliente final, sean o no sean regulados deberá garantizar que al menos el $10 \%$ de la generación proviene de fuentes renovables no convencionales.

\section{Discusión}

Como se puede observar hay una gran congruencia en el sector eléctrico de Centroamérica, ya que todos los países comprendidos por esta región están apostando en una misma dirección: buscan la diversificación de su matriz de generación ampliando el aporte de las fuentes de energía no convencionales de energía, como lo pueden ser las centrales fotovoltaicas. Además, para esta región continental y de habla hispana que comprende los países desde México hasta Panamá, el marco legal y regulatorio es muy afín, y con una perspectiva clara, impulsando el uso de estas fuentes mediante incentivos, lo que invita la inversión extranjera: de esta misma parte la apertura a agentes privados a la participación de este proceso. Esta motivación por medio de incentivos es de uso generalizado en centro américa y cada país cuenta con sus propias entidades estatales que regulan y evalúan los proyectos que están sujetos a estos beneficios. Otra diferencia que se encuentra en esta región, es la determinación del límite de tamaño para los generadores. También se resalta el caso panameño, el cuál es el único país de la región que tiene una ley específica para centrales fotovoltaicas.

Por su parte, la región suramericana no tiene un marco regulatorio fuerte con respecto a las fuentes de energía renovables no convencionales como se evidencia en la tabla anterior, aunque en algunos casos se pueda aseverar que la intención de que estas fuentes ingresen en su matriz energética sea más fuerte y de concepto más amplio que en otros. 


\section{Observacion del contexto colombiano}

Haciendo una comparación y observación de las normas, incentivos y marcos regulatorios de la Generación de Energía Eléctrica por medio de fuentes Renovables No Convencionales en los países de habla hispana de Latinoamérica, con la situación actual en el marco regulatorio de Colombia se pueden establecer ciertos criterios comparativos del país con el contexto general de la región. En primer lugar, se observa como punto general que los países de la parte central de Latinoamérica comparten el mismo contexto para los incentivos y políticas para el uso de las Energías Renovables: Guatemala, El Salvador, Nicaragua, Costa Rica y Panamá en sus Leyes correspondientes decretan un artículo para los incentivos como exoneraciones de impuestos como del IVA, o los de importación de equipos y maquinaria con fines para el uso de esta tecnología entre otros. México por su parte en su Ley no específica incentivos, pero sin embargo si especifica la creación del Fondo para la transición Energética y el Aprovechamiento Sustentable de la Energía con el propósito de potenciar el financiamiento e inversión en las energías limpias.

Por otro parte, en cuanto a las limitaciones de capacidad para las centrales solares fotovoltaicas y/o parques solares fotovoltaicos se establece en la mayoría un límite de capacidad de 20MW, con algunas excepciones como México, Guatemala, y El Salvador en donde no se éstos establecen límites de capacidad.

En cuanto a América del Sur de hispana existe cierta particularidad, pues se puede observar que pocos países de este sector están involucrados totalmente con el tema. Tal es el caso de Argentina, Chile y Uruguay, que en sus marcos regulatorios describen de una forma más explícita todo lo relacionado con la regulación de las Energías Renovables y son los únicos que establecen incentivos para el uso y aplicación de la generación de energía eléctrica por medio de éstas. Aunque no son del todo específicas, se mencionan ciertos incentivos como la exención de impuestos de IVA, e impuestos a las ganancias, entre otras. Adicionalmente, las regulaciones de estos países, son las únicos que mencionan si existe o no una limitación de capacidad para las centrales solares fotovoltaicas: en Chile no se estable un límite de capacidad y Uruguay establece unas franjas limites hasta 50MW. En el caso opuesto, Bolivia y Venezuela carecen de normativas y leyes para la producción de energía eléctrica por medio de energías alternativas.

En cuanto a la situación actual de Colombia se puede mencionar que existe la Ley 1715 [126], por medio de la cual se regula la integración de las energías renovables no convencionales al sistema energético nacional, la cual entró en vigencia el 13 de Mayo de 2014. Tiene por objetivo "promover el desarrollo y la utilización de las fuentes no convencionales de energía, principalmente aquellas de carácter renovable, en el sistema energético nacional, mediante su integración al mercado eléctrico, su participación en las zonas no interconectadas y en otros usos energéticos como medio necesario para el desarrollo económico sostenible".

La ley se estipulan las competencias administrativas de las entidades del gobierno como el Ministerio de Minas y Energía, la Comisión de Regulación de Energía y Gas (CREG), el Ministerio de Hacienda y Crédito, entre otras, para la promoción de los proyectos con FNCE, cada una de estas con funciones 
específicas. También se resalta resaltar una intención del uso de las FNCER para generación a pequeña escala, y una política de instrumentos tributarios de promoción dentro de éste tipo de proyectos, donde se destacan la deducción de hasta el $50 \%$ del valor de los proyectos vía el impuesto de renta y depreciación acelerada de Activos. En cuanto a la Generación Solar Fotovoltaica, el Artículo 19 habla en específico de éste sector.

Es importante resaltar que a la efectividad de estas propuestas establecidas en la ley dependerá en gran medida de la reglamentación técnica que se desarrolle por la CREG, la cual a la fecha aún sigue pendiente.

Por otro lado surgen opiniones y recomendaciones de los agentes del mercado con respecto a la ley las cuales no se puede dejar de lado, por ejemplo algunas de sus recomendaciones son [127]:

- Tener más claridad en cuáles son las fuentes renovables a promover, que incentivos tendría cada una y cuál sería el mecanismo de priorización entre ellas.

- En la parte de incentivos, no establecer un límite de reducción de renta ya que la inversión en equipos para FNCE puede ser muy alta y esto podría desestimular la inversión; por otro lado el beneficio de exclusión del IVA debe ser más general, ya que al manejar una lista en específico, se limita las características propias de un proyecto de investigación y desarrollo.

De acuerdo con lo anterior, se observa que la intención central de Ley 1715 de 2014, no difiere mucho en cuanto a las normativas regulatorias que se encontraron en los países de habla hispana en Latinoamérica, que ya tienen alguna historia en el fomento y motivación a proyectos con energías renovables, enfocados a la generación de electricidad con energía solar fotovoltaica. En la mayoría de estos países, lo que se puede ver en común es la participación tanto del gobierno como de entidades privadas, el apoyo de organizaciones internacionales y reglas claras en lo relacionado con los incentivos, procedimientos, adecuaciones, límites y condiciones afines para las fuentes de energía renovables. Por su parte en Colombia, a pesar de haberse cumplido el plazo especificado, a la fecha todavía no existe la reglamentación que permita conocer claramente las condiciones para el mercado fotovoltaico. Entre estas condiciones desconocidas se destacan la ausencia de algún límite en KW o MW para grandes proyectos, la forma de acceso a los incentivos tributarios y los escenarios de mercados para los posibles excedentes de energía para usuarios auto generadores. Adicionalmente, se observa que el enfoque de dicha ley está dado únicamente a proyectos residenciales, comerciales e industriales y no a generadores exclusivos es decir, tampoco se conoce aún si los proyectos privados que generen energía eléctrica por medio de energía renovable no convencional, podrán aportar energía a la red de transmisión y distribución. Por otra parte, tampoco se fijan metas para que en un tiempo determinado la generación de electricidad sea cien por ciento $(100 \%)$ con fuentes de energía renovables, como si lo indican muchas de las normativas de otros países de habla hispana en Latinoamérica.

Finalmente, es de destacar que en Colombia existe un gran territorio nacional que no se encuentra interconectado a la red nacional de electricidad, zonas 
conocidas como ZNI (Zonas No Interconectadas) y uno de los objetivos que persigue esta Ley es dar energía a éstos sitios ya sea por medio de fuentes no convencionales. Sin embargo, aún no es claro cómo se va a realizar ésta tarea, porque como se mencionó anteriormente, no se habla de proyectos a mediana o gran escala, que puedan contribuir a que estas zonas lleguen a tener la prestación del servicio.

\section{Conclusiones}

Las energías renovables son un punto de fuerte potencial para el desarrollo de la nueva era de los países, en cuanto a los ámbitos social, político, económico y ambiental. En una era en donde se han visto todos los efectos que ha causado el cambio climático por culpa de los gases de efecto invernadero, y del uso ineficiente e irracional de la energía, es necesario aprovechar al máximo los recursos que brinda el suelo, el aire, el agua, el sol, etc., como recursos de fuentes de energía renovables no convencionales, para la generación de energía eléctrica.

Es de gran importancia e indispensable que el gobierno, conformado por sus diferentes entes para el control, regulación y creación de leyes, haga presencia y sea quien tome la iniciativa de buscar alternativas para que se incentive el uso de estas energías limpias para la generación de energía eléctrica, a través de una regulación efectiva de la ley 1715 .

Como se ha observado, las tecnologías limpias y renovables, no son tan nuevas como se cree en el continente latinoamericano. La mayoría estos países han venido trabajando desde hace tiempo en sus marcos regulatorios, y han implementado proyectos y directrices propias y en algunas casos como región, tal como se puede ver en similitud de las políticas en el continente centroamericano, la cual es la zona donde se ha podido observar el mayor crecimiento de centrales fotovoltaicas, a través una constante inversión tanto pública como privada. Sin embargo, es evidente que se observa que aún falta un largo camino para cumplir la meta de que la generación de energía eléctrica sea del $100 \%$ con recursos renovables. Ya que Centroamérica fue visto como región y sus políticas van ligadas y estructuradas de esa manera, se encuentra un gran contraste con la parte de países en éste estudio pertenecientes a Sur América, en donde sí se buscan objetivos y metas en cuanto a generación de energía eléctrica mediante recursos renovables, pero de forma muy particular, donde cada país va en una propia dirección. Este contexto diverso en cuanto a políticas y marco regulatorio, bien podría ser una de las causas del poco progreso en ésta región en cuanto al tema en donde se resaltan falencias en diversos aspectos como en la infraestructura, o en la parte regulatoria y de incentivos promotores de estas fuentes.

En el contexto colombiano, existe la ley 1715 de 2014, en la que se establece el uso de recursos renovables no convencionales para la generación de energía eléctrica, y en donde aparecen una serie de incentivos, como exenciones fiscales, entre otros. Sin embargo, es vital que se establezca su reglamentación, ya que sin ésta será imposible desarrollar el sector fotovoltaico tal como se está haciendo en los países vecinos. Adicionalmente, esta reglamentación deberá evidenciar una 
clara aplicación para proyectos a gran escala, como Parques Solares Fotovoltaicos, ya que como está actualmente formulada la ley, se observa un enfoque solo a proyectos pequeños para el uso residencial, comercial e industrial como auto generadores para su propio consumo. Es evidente que por algo se debe iniciar, pero la meta, aprovechando al máximo los recursos naturales que Colombia tiene, debería ser llegar a que el $100 \%$ de la energía eléctrica que se consuma, sea producida por recursos de energía renovables no convencionales. Por esta misma razón, se debería ampliar la cobertura para que la aplicación de esta Ley, acoja tanto proyectos pequeños como proyectos de gran escala.

\section{Referencias}

1. REN21 (2014). Renewables 2014 Global Status Report.

2. Grupo Intergubernamental de Expertos sobre el Cambio Climático (2011). Fuentes de Energía Renovables y Mitigación Del Cambio Climático.

3. Naciones Unidas (1998). Protocolo De Kyoto De La Convención Marco De Las Naciones Unidas Sobre El Cambio Climático.

4. Naciones Unidas (1992). Declaración de Rio sobre el Medio Ambiente y Desarrollo.

5. IRENA (2014). The Socio-economic Benefits of Solar and Wind Energy.

6. IRENA (2014). RENEWABLE POWER GENERATION COSTS IN 2014.

7. BNEF (Bloomberg New Energy Finance) (2014). Q4 2014 Global PV Market Outlook, BNEF, London.

8. IRENA (2015). Renewable Energy in Latin America 2015: An Overview of Policies.

9. H.D. Kambezidis (2012). Reference Module in Earth Systems and Environmental Sciences, from Comprehensive Renewable Energy, Volume 3, 2012, Pages 27-84, Current as of 20 April 2015.

10. Dr. Prof. Arq. John Martin Evans (2010). "Hábitat y Energía" Jornada: Energías Renovables para la Ciudad de Buenos Aires en el marco del Cambio Climático. Defensoría del Pueblo, Buenos Aires.

11. Reinhold Schmidt (2008). "Curso Energía Fotovoltaica Aspectos técnicos y aplicaciones". Centro Tecnológico Nuevos Horizontes, Chile.

12. CIE (Corporación para la Investigación Energética), CONELEC. ATLAS SOLAR DEL ECUADOR CON FINES DE GENERACIÓN ELÉCTRICA.

13. Ing. Químico Víctor Herbert De León Morales (2008). "Generación Eléctrica Fotovoltaica En La Facultad De Ingeniería Usac Y Estudio Del Aprovechamiento" M.S Tesis, Universidad De San Carlos De Guatemala.

14. José Manuel Flores (2006). "Estado Actual De La Energia Solar En Honduras: Investigación Exploratoria", Universidad Nacional Autónoma De Honduras.

15. Ing. Ricardo Saldaña Flores, Fís. Ubaldo Miranda Miranda (2005). "Estudio De Los Potenciales Bioenergético, Eólico, Minihidráulico Y Solar En México".

16. Carlos Martinez Catedra (2007). "Estudios de Necesidades y Recursos Energéticos de Comunidades Aisladas en Nicaragua". Escola Técnica Superior d'Enginyeria Industrial de Barcelona.

17. Servicio Nacional De Meteorología E Hidrología (2004). "Atlas de Radiación Solar Perú" 2004.

18. Mauricio Ayala, Ismael Sánchez, Arturo Escalante, Willian Marroquín (2005). "Determinación del potencial solar y eólico en El Salvador" Ministerio de Medio Ambiente y Recursos naturales (MARN), Universidad Centroamericana "José Simeón Cañas" (UCA), Servicio Nacional de Estudios Territoriales (SNET/MARN). 
19. Dr. Gonzalo Abal (2010). "Estimación de la irradiancia solar diaria promedio en Uruguay" Universidad de la República Montevideo, Uruguay.

20. Fausto Posso, Juan Herranz (2011). "Estudio Sobre La Utilización De La Energía Solar Fotovoltaica En Hogares Rurales De Venezuela" Caracas.

21. Ministerio de Economía y Finanzas, República de Panamá (2001). "Sistemas Solares Fabricados e Instalados en América Central".

22. Marcelo J. Lucano, Iván E. Fuentes (2010). "Evaluación Del Potencial De Radiación Solar Global En El Departamento De Cochabamba Utilizando Modelos De Sistemas De Información Geográfica e Imágenes Satelitales" Revista Boliviana De Física 16, $13 \hat{a ̂ A} \widehat{2} 21$.

23. Viceministerio de Minas y Energía (2013). Radiación Solar Promedio. Ande 2013.

24. UPME. Atlas de Radiación Solar de Colombia.

25. SENER (Secretaria de Energía) (2013). Prospectiva del Sector Eléctrico 2013-2027.

26. Gilberto Alfaro, David Jiménez, Arturo Saavedra (2014). "La Reforma Energética y el Sector Eléctrico en México" KPMG.

27. Ramos Gutiérrez Leonardo De Jesús, Montenegro Fragoso Manuel (2012). "La Generación De Energía Eléctrica En México" XXII Congreso Nacional De Hidráulica, Acapulco, Guerrero, México, Noviembre 2012.

28. SE (Secretaria de Economía) Energías Renovables, Unidad de Inteligencia de Negocios. 2013.

29. Ley para el aprovechamiento de energías renovables y el financiamiento de la transición energética. México.

30. Arianna Galan (2015). "Aura Solar I Photovoltaic Plant, Mexico" Zofnass Program for Sustainable Infrastucture 2015.

31. Comisión Reguladora de Energía. "RESOLUCIÓN Núm. RES/004/2015".

32. PV Magazine "La segunda mayor central solar de México inaugurada en Durango" publicación 30/05/14. Obtenida en Julio 2015 de http://www.pv-magazine-latam.com/noticias/detalles/articulo/ la-segunda-mayor-central-solar-de-mxico-inaugurada-en-durango_ $100015481 /$.

33. PV Magazine "La mexicana CFE sostiene que la reforma energética favorecerá a las renovables" publicación 28/04/14. Obtenida en Julio 2015 de http://www.pv-magazine-latam.com/noticias/detalles/articulo/ la-mexicana-cfe-sostiene-que-la-reforma-energtica-favorecer-a-las/ -renovables_100015099/.

34. Instituto de Investigaciones Eléctricas, GEF (Global Enviroment Facility). "Parque Solar Bicentenario Green Growth" 3er Coloquio Internacional Sistemas Fotovoltaicos.

35. RED SolLAC "Central Solar Fotovoltaica Piloto Santa Rosalia". Obtenida en Julio 2015 de http://redsollac.org/nuevo/ mexico-1mw-central-solar-fotovoltaica-piloto-santa-rosalia-2/.

36. Ley de Incentivos para el desarrollo de Proyectos de Energía Renovable. Guatemala.

37. Proyecto ARECA. "Análisis del Mercado Guatemanteco de Energía Renovable" 2009.

38. BNAMERICAS "Planta Solar Fotovoltaica Horus I". Obtenida en Julio 2015 de http://www.bnamericas.com/project-profile/es/ horus-i-solar-plant-horus-i.

39. PV Magazine "Guatemala acoge una Central Solar de 5MW, La Mayor de Centroamerica" publicación 22/04/14. Obtenida en Julio 2015 de http://www.pv-magazine-latam.com/noticias/detalles/articulo/ 
guatemala-acoge-una-central-solar-de-5-mw--la-mayor-de-centroamrica_ $100014972 /$.

40. Proyecto ARECA. "Análisis del Mercado Salvadoreño de Energía Renovable" 2010.

41. Ley 462: Ley de Incentivos Fiscales para el Fomento de las Energías Renovables en la Generación de Electricidad. El Salvador.

42. Acuerdo No 162 - E-2012: Normativa Técnica para Caracterizar los Proyectos que Aprovechan las Fuentes Renovables en la Generación de Energía Eléctrica.

43. SMA. "Planta Fotovoltaica en Atacama en operación con tecnología SMA" Nota de Prensa. Junio 2014.

44. SUNPOWER. "Total y SunPower celebran la apertura de la Planta Solar PV Salvador de 70 MW" Nota de Prensa. Enero 2015.

45. Proyecto ARECA. "Análisis del Mercado Hondureño de Energía Renovable" 2009.

46. Planta Solar Pavana. Obtenida en Julio 2015 de http://www.hondudiario.com/ ?q=node $/ 17305$.

47. Planta Solar Marcovia. Obtenida en Julio 2015 de http://www. bnamericas.com/ project-profile/es/marcovia-solar-plant-marcovia.

48. PV Magazine "Honduras: Corporación Aura construye central de 61 MW" publicación 16/12/14. Obtenida en Octubre 2015 de http://www.pv-magazine-latam.com/noticias/detalles/articulo/ honduras--corporacin-aura-solar-construye-central-de-61-mw_ $100017676 /$.

49. PV Magazine "Honduras: La Noruega Scatec asegura Financiación para central solar de 60 MW" publicación 30/12/14. Obtenida en Octubre 2015 de http://www.pv-magazine-latam.com/noticias/detalles/articulo/ honduras--la-noruega-scatec-asegura-financiacin-para-central-solar/ -de-60-mw-_100017760/.

50. PV Magazine "Honduras: Canadian Solar suministra módulos para proyectos FV con 146 MW" publicación 15/10/14. Obtenida en Octubre 2015 de http://www.pv-magazine-latam.com/noticias/detalles/articulo/ honduras--canadian-solar-suministra-mdulos-para-proyectos-fv-con/ -146-mw_100016907/

51. SOPOSA-COHESSA. Obtenida en Octubre 2015 de http://soposa.com/

52. PV Magazine "Honduras: Central solar $70 \mathrm{MW}$ entrara en servicio antes del 1 de agosto" publicación 23/07/15. Obtenida en Octubre 2015 de http://www.pv-magazine-latam.com/noticias/detalles/articulo/ honduras--central-solar-de-70-mw-entrar-en-servicio-antes-del-1-de/ -agosto_100019956/.

53. La Gaceta, República de Honduras Decreto 267-98. Obtenida en Octubre 2015 de https://docs.google.com/viewer?url=http://faolex.fao.org/docs/pdf/ hon92280.pdf.

54. La Gaceta, República de Honduras, Decreto 70-2007. Obtenida en Octubre 2015 de https://docs.google.com/viewer?url=http://www. poderjudicial.gob. $\mathrm{hn} /$ juris/Leyes/Ley $\backslash \% 20$ de $\ \% 20$ Promocion $\backslash \% 20 \mathrm{a} \backslash \% 201 \mathrm{a} \backslash \% 20$ Generacion $\backslash \% 20 \mathrm{de} \backslash$ $\% 20$ Energia $\ \% 20$ Electrica $\ \% 20$ con $\backslash \% 20$ Recursos $\backslash \% 20$ Renovables.pdf.

55. La Gaceta, República de Honduras, Decreto 404-2014. Obtenida en Octubre 2015 de https://docs.google.com/viewer?url=http://www.enee.hn/noticias/ Ley $\backslash \% 20$ General $\backslash \% 20$ de $\backslash \% 201$ a $\backslash \% 20$ Industria $\backslash \% 20$ Electrica $\ \% 20$ Honduras $\backslash$ $\% 20-\backslash \% 20$ Decreto $\% 20404-2014$.pdf.

56. Proyecto ARECA (2010). "Análisis del Mercado Nicaragüense de Energía Renovable". 
57. IRENA (2015). "Nicaragua: EVALUACIÓN DEL ESTADO DE PREPARACION DE LAS ENERGÓAS RENOVABLES" Enero 2015.

58. Ley 532 "Para La Promoción De Generación Eléctrica Con Fuentes Renovables". Nicaragua.

59. REVE "Nicaragua Inaugura central de energía Fotovoltaica de 1,38 MW", publicación 22/02/13. Obtenida en Octubre 2015 de http: //www.evwind.com/2013/02/22/nicaragua-inaugura-central-de-energia/ -solar-fotovoltaica-de-138-mw/.

60. ICE (Instituto Costarricense de Electricidad) (2014). Plan de Expansión de la Generación Eléctrica periodo 2014-2035.

61. José María Blanco (2013). BID (Banco Interamericano de Desarrollo). "Sustainable Energy for all, Evaluación Rápida y Análisis de Vacíos Costa Rica" Marzo 2013.

62. Ley 7200 "Generación Eléctrica Paralela O Autónoma". Costa Rica.

63. ARESERP: "ARESEP propone tarifas para promover la generación solar", Obtenida en Octubre 2015 de http://aresep.go.cr/usuarios/noticias/ 1168-aresep-propone-tarifas-para-promover-la-generacion-solar.

64. SmartGrid Costa Rica "Arranca Parque Solar Miravalles Y Proyecto Solar Ice Sabana" Publicación 22/11/12. Obtenida en Julio 2015 de http://www.smartgridcostarica.com/2012/11/21/arranca-parque/ -solar-miravalles-y-proyecto-solar-ice-sabana/.

65. Ing. Roberto Matheus (2010). "Descripción del Sector Eléctrico de Panamá" Universidad Tecnológica de Panamá, Facultad de Ingeniería Eléctrica, Agosto 2010.

66. Proyecto ARECA (2010). "Análisis del Mercado Panameño de Energía Renovable". 67. ETESA (2014). Plan de Expansión del Sistema Interconectado Nacional 2014-2028.

68. La Ley 37 "Establece El Régimen De Incentivos Para El Fomento De La Construcción, Operación Y Mantenimiento De Centrales Y/O Instalaciones Solares". Panamá.

69. Ing. Fernando Díaz. Director de Electricidad. Secretaria Nacional de Energía. xtenda. "Jornada online Canal Renovables Centroamérica". Mayo 2015.

70. Fabio Klitenik, Pablo Mira, Pablo Moldovan(2009). "El Mercado Eléctrico Argentino" Ministerio de Economía y Finanzas Publicas. Cuarto Trimestre 2009.

71. CNEA (Comisión Nacional de Energía Atómica) (2015). "Síntesis del Mercado eléctrico Mayorista de la República Argentina" Ministerio de Planificación Federal, Inversión Pública y Servicios. Marzo 2015.

72. Santiago Garrido (2013). "Energías renovables y dinámicas de desarrollo en Argentina: Políticas de universalización del acceso y diversificación de la matriz energética." Conferencia Internacional LALICS, Rio de Janeiro, Brasil. Noviembre 2013.

73. Ley 26.190 "Régimen De Fomento Nacional Para El Uso De Fuentes Renovables De Energía Destinada A La Producción De Energía Eléctrica”. Argentina.

74. PV Magazine "Argentina: Mendoza acogerá central solar de 1 MW" publicación 22/04/15. Obtenida en Julio 2015 de http://www.pv-magazine-latam.com/noticias/detalles/articulo/ argentina--mendoza-acoger-central-solar-de-1-mw_100018969/.

75. D.H. Pontoriero, Marcelo Gustavo Molina (2009). "Evaluación Técnica Y Económica De Generación Híbrida Solar-Eólica En La Provincia De San Juan - Argentina" Décimo Tercer Encuentro Regional Iberoamericano De Cigré Argentina. Mayo 2009. 
76. RED SolLAC "Argentina, 5 MW, Parque Solar Cañada Honda". Obtenida en Julio 2015 de http://redsollac.org/nuevo/argentina/ -5mw-parque-solar-canada-honda/.

77. PV Magazine "Argentina: Se inagura el primer parque FV de la Provincia de San Luis" publicación 21/10/14. Obtenida en Julio 2015 de http://www.pv-magazine-latam.com/noticias/detalles/articulo/ argentina--se-inaugura-el-primer-parque-fv-de-la-provincia-de/ -san-luis_100016975/.

78. IRENA (2014). PERU EVALUACION DEL ESTADO PREPARACION DE LAS ENERGIAS RENOVABLES 2014.

79. Decreto Legislativo 1002 Promoción de Generación con uso de Energías Renovables. Perú. Disponible en https://docs.google.com/viewer?url=http: //www.minem.gob.pe/archivos/legislacion-9ozj22z9ap5zz33z-DL_de_ promocion_de_la_inversion_para_la_generacion_de_electricidad_con_ el_uso_de_energias_renovables_1002.pdf.

80. Decreto Supremo 050-2008-EM. Perú. Obtenida en Octubre 2015 de https://docs.google.com/viewer?url=http://www.coes.org.pe/dataweb3/ $2010 / \mathrm{djr} /$ baselegal/Aprueban $\ \% 20$ Reglamento $\ \% 20 \mathrm{de} \backslash \% 201 \mathrm{a} \backslash \% 20$ Generacion $\backslash$ $\% 20$ de $\backslash \% 20$ Electricidad $\backslash \% 20$ con $\backslash \% 20$ Energias $\backslash \% 20$ Renovables.pdf.

81. Norma Técnica Peruana. NTP 399.403.2006.

82. Panamerica Solar. Obtenida en Julio 2015 de http://www.gestampsolar.com/es/ negocio/proyectos-de-investigacion/panamericana-solar.

83. Tacna Solar. Obtenida en Julio 2015 de http://www.gestampsolar.com/es/ negocio/proyectos-de-investigacion/tacna-solar-0

84. Majes solar. Obtenida en Marzo 2015 de http://www.bnamericas.com/ project-profile/es/majes-solar-20-t-solar-plant-majes-solar-20-t.

85. Planta Solar Arepa. Obtenida en Agosto 2015 de http://www. bnamericas.com/ project-profile/energiaelectrica/arepa-solar-plant-arepa

86. Planta Solar La Joya. Obtenida en Agosto 2015 de http://www.bnamericas.com/ project-profile/energiaelectrica/la-joya-solar-plant-la-joya

87. Central Solar Pampa Colorada. Obtenida en Agosto 2015 de http://www.bnamericas.com/project-profile/energiaelectrica/ pampa-colrada-solar-plant-pampa-colorada

88. Central Solar Pampa Las Pulgas. Obtenida en Agosto 2015 de http://www.bnamericas.com/project-profile/energiaelectrica/ pampa-las-pulgas-pampa-las-pulgas

89. Central Solar Pampa Siguas. Obtenida en Agosto 2015 de http://www.bnamericas.com/project-profile/energiaelectrica/ pampa-siguas-solar-plant-pampa-siguas

90. Central Solar Tesoro. Obtenida en Agosto 2015 de http://www. bnamericas.com/ project-profile/energiaelectrica/tesoro-solar-plant-tesoro

91. CONELEC (2011). Estadística del Sector Eléctrico Ecuatoriano. Folleto Multianual 2011.

92. Paula García Zaragoza (2011). "El Mercado de las Energías Renovables en Ecuador". ICEX (Instituto Español de Comercio Exterior).

93. Planta Solar Fotovoltaica Santa Elena. Obtenida en Julio 2015 de http://www.bnamericas.com/project-profile/es/santa-elena/ -photovoltaic-plant-santa-elena.

94. Aguilar, C.; Galarza, L.; Recalde, A (2015). "Mejoramiento de la Calidad del Producto Técnico e Impacto en la Utilización de Generación Distribuida con 
Energías Renovables en una Zona de la provincia de Manabí.”. Revista Técnica Energía. Enero 2015, Issue 11, p92-100. 9p.

95. Ing. Damián Lara Salazar.Gran Solar (2013). "Ficha Ambiental para la Línea de Transmisión a 13.8Kv Asociada al Proyecto Fotovoltaico Salinas". Octubre 2013.

96. Dr. Ing. Gonzalo Casaravilla (2011). "Infraestructura Energética del Sector Eléctrico 2010-2020." Revista Técnica Ingeniería. Diciembre 2011. P26-31.

97. Diego Velázquez Terranoba (2012). "El Mercado de las Energías Renovables en Ecuador". ICEX (Instituto Español de Comercio Exterior). Mayo 2012.

98. Uruguay XXI Promoción de Inversiones y Exportaciones (2014). "Energías Renovables: Oportunidad de Inversión" Agosto 2014.

99. Oficina Económica y Comercial de la Embajada de España en Montevideo. ICEX (2012). "El Sector de las Energías Renovables en Uruguay". Mayo 2012.

100. Ley 16.906 de Promoción y Protección de Inversiones. Uruguay.

101. BID. "Proyecto Energía Solar La Jacinta". Obtenida en Julio 2015 de http://www.iadb.org/es/proyectos/project-information-page, 1303.html? id=UR-L1092.

102. Ley NÂr 1604 de Electricidad de 1994. Disponible en https://docs. google.com/viewer?url=http $\ \% 3 \mathrm{~A} \backslash \% 2 \mathrm{~F} \backslash \% 2 \mathrm{Fwww}$. hidrocarburos.gob.bo $\backslash$ $\% 2$ Fphocadownload $\ \% 2$ FLey $\backslash \% 2520$ de $\backslash \% 2520$ Electricidad.pdf

103. BID (2013). Evaluación Financiera Y Económica Del Proyecto Eterificación Rural Con Energía Renovable (BO-X1013). Julio 2013.

104. PV Magazine "Bolivia abre licitación del proyecto solar de $5 \mathrm{MW}$ en Pando" publicación 19/03/14. Obtenida en Octubre 2015 de http://www.pv-magazine-latam.com/noticias/detalles/articulo/ bolivia-abre-la-licitacin-del-proyecto-solar-de-5-mw-en-pando_ 100014555/

105. Administración Nacional de Electricidad "ANDE". Obtenida en Octubre 2015 de http://www.ande.gov.py/

106. OLADE (2003). La Situación Energética en América Latina Informe Final. Marzo 2003.

107. Ley 3009 de la Producción y Transporte Independiente de Energía Eléctrica. Disponible en https://docs.google.com/viewer?url=http://www.ssme.gov . py/vmme/pdf/leyes/Ley $\backslash 203009 \backslash \% 20$ PTIEE. pdf.

108. Desarrollo de las Energías Renovables en Venezuela. Obtenida en Agosto 2015 de http://latinoamericarenovable.com/2013/06/29/ el-desarrollo-de-las-energias-renovables-en-venezuela-reto-o-utopia/.

109. FUNDELEC (2007). "La Luz del Alba: Energías Renovables por un Futuro Mejor". Edición: Enero- Marzo 2007.

110. Empresa de nacional de electricidad sociedad anónima chile. Habilitado: http: //www.endesa.cl/es/Paginas/home.aspx

111. Sistema interconectado del norte grande. Obtenida en Octubre 2015 de http://antiguo.minenergia.cl/minwww/opencms/03_Energias/Otros_ Niveles/Electricidad/Sistema_Electrico/sing.html

112. Sistema interconectado central. Obtenida en Octubre 2015 de http://www. cdecsic.cl/sobre-cdec-sic/sic/

113. Diario oficial de la república de Chile. Obtenida en Septiembre 2015 de https://docs.google.com/viewer?url=http://antiguo.minenergia.cl/ minwww/export/sites/default/08_Normativas/02_energias/descargable_ renovables/2008_ResEx1278_ERNC.pdf

114. Ley 20257 Ley general de servicios eléctricos respecto de la generación de energía eléctrica con fuentes de energías renovables no convencionales. 
115. Proyecto solar RAVSOE. Obtenida en Octubre 2015 de http://www.bnamericas.com/en/project-profile/electricpower/ planta-solar-fotovoltaica-ravsoe-ravsoe?idioma=en

116. Proyecto solar Pampa solar norte PV. Obtenida en Octubre 2015 de http://www.bnamericas.com/en/project-profile/electricpower/ pampa-solar-norte-pv-solar-project-pampa-solar-norte?idioma=en

117. Proyecto el Tamarugo Dólar Granja. Obtenida en Octubre 2015 de http://www.bnamericas.com/en/project-profile/electricpower/ parque-solar-el-tamarugo-el-tamarugo?idioma=en

118. Proyecto El Chañar Solar. Obtenida en Octubre 2015 de http://www.bnamericas.com/en/project-profile/electricpower/ parque-solar-el-chanar-el-chanar?idioma=en

119. Proyecto Fotovoltaico Laberinto Solar. Obtenida en Octubre 2015 de http://www.bnamericas.com/en/project-profile/ electricpower/bolero-oeste-solar-photovoltaic-project/ -ex-laberinto-bolero-oeste-ex-laberinto?idioma=en

120. Proyecto solar Sol del Loa. Obtenida en Octubre 2015 de http://www.bnamericas.com/en/project-profile/electricpower/ sol-del-loa-solar-plant-sol-del-loa?idioma=en

121. Proyecto solar Chaka PV Planta solar. Obtenida en Octubre 2015 de http://www.bnamericas.com/en/project-profile/electricpower/ chaka-pv-solar-plant-chaka?idioma=en

122. Complejo solar FV Pica. Obtenida en Octubre 2015 de http://www.bnamericas.com/en/project-profile/electricpower/ fv-pica-solar-complex-complejo-solar-fv-pica?idioma=en

123. Huerta solar Fotovoltaica Planta. Obtenida en Octubre 2015 de http://www.bnamericas.com/en/project-profile/electricpower/ huerta-solar-photovoltaic-plant-huerta-solar-fotovoltaica?idioma=en

124. Proyecto fotovoltaico Maria Elena. Obtenida en Octubre 2015 de http://www.bnamericas.com/en/project-profile/electricpower/ parque-fotovoltaico-maria-elena-fotovoltaico-maria-elena?idioma=en

125. Planta solar Amanecer. Obtenida en Octubre 2015 de http://berc.berkeley. edu/atacama-the-sun-of-chilean-energy/?utm_source=BERC+Master+List\& utm_campaign=3ec5c53ad2-Weekly_BERC_1_Sep_269_26_2011\&utm_medium= email\&utm_term=0_6307259a70-3ec5c53ad2-277409841

126. Ley 1715 de 2014 "Por Medio De La Cual Se Regula La Integración De Las Energías Renovables No Convencionales Al Sistema Energético Nacional".

127. Ministerio de Minas. Grupo de Participación y Servicio al ciudadano: Informe Documento en Discusión: Proyecto de Decreto "Por el cual se establecen los lineamientos de política en materia de la aplicación de los incentivos a la inversión en proyectos de fuentes no convencionales de energía y gestión eficiente de la energía contemplados en el Capítulo III de la Ley 1715 de 2014." (Abril 2015). Obtenida en Octubre 2015 de https://www.minminas. gov.co/documents/10180/1186017/InformeFuentesNoConvencionalesweb.pdf / $5854942 b-f c 40-4457-8 a 09-1 a b 0928 a b 80 c$ 